\title{
Video-Assisted Anterior Retroperitoneal Approach to the Lumbar Spine. A Minimal Invasive Technique Improved by the Use of Endoscopic Camera to Treat Lumbar Spine Diseases
}

\author{
Roberto Bassani1, Riccardo Cecchinato', Carlotta Morselli' ${ }^{2}$ Pedro Berjano, \\ Claudio Lamartina ${ }^{1}$ \\ ${ }^{1}$ IRCCS Galeazzi Orthopedic Institute, Milan, Italy \\ ${ }^{2}$ Department of Neurosurgery and Gamma Knife Radiosurgery, San Raffaele Hospital, Vita-Salute University, \\ Milan, Italy \\ Email: r.bassani.spine@gmail.com
}

Received 30 October 2015; accepted 26 January 2016; published 29 January 2016

Copyright (C) 2016 by authors and Scientific Research Publishing Inc.

This work is licensed under the Creative Commons Attribution International License (CC BY). http://creativecommons.org/licenses/by/4.0/

(c) (i) 0pen Access

\begin{abstract}
The anterior retroperitoneal approach is a technique to treat disc degenerative disease (DDD), spinal deformities, traumas, tumors and infections. It can be used to perform Anterior Lumbar Interbody Fusions (ALIF) or Total Disc Replacements (TDR). Though being a fast procedure that is becoming more often used by spinal surgeons, the anterior approach requires an adequate knowledge of the anatomy of the abdomen to lessen the risks of intraoperative complications. The authors' preferred technique is the left retroperitoneal video-assisted approach, using an endoscope to visualize and magnify the deep anatomical structures, discectomy procedure and hardware implant. In a review of our casuistry from 2010 to 2012, 163 patients underwent an anterior lumbar approach, and 139 of these had a single L4-5 or L5-S1 level treatment. A 3.6\% rate of global complications $(1.44 \%$ of major vascular injuries) has been observed, with a mean operation time of 116.4 minutes and a mean blood loss of $156.1 \mathrm{ml}$. These values show how the anterior retroperitoneal video-assisted approach for the treatment of lumbar diseases is a fast procedure that implies low blood loss, with a low rate of intraoperative complications.
\end{abstract}

\section{Keywords}

Video-Assisted Anterior Retroperitoneal Approach, ALIF, TDR 


\section{Introduction}

An anterior approach to the lumbar spine is often required in patients affected by chronic low back pain with or without radiculopathy, non-responsive to of conservative treatment (medication, physical and pain therapy) for a period of at least 6 months. Degenerative disc disease (DDD), degenerative spondylolisthesis, lumbar stenosis with segmental instability, previous unsuccessful posterior lumbar surgery, non-union, restoration of disc height, spinal deformities, trauma or infections are the main indications for anterior lumbar approach.

On the other hand, the main challenges are previous abdominal or gynecological surgery performed by the retroperitoneal or transperitoneal routes (colonic resection, hysterectomy), vascular anatomical variations (low iliac bifurcation: in front of L5-S1, extremely lateral path of the common iliac vein on the left side: laterally in the L4-L5 space) and abdominal diseases (Crohn's disease, ulcerative colitis).

There are different kinds of anterior approaches described in the literature: the eXtreme Lumbar Interbody Fusion (XLIF) is a modification more recently introduced, which involves a lateral incision, a blunt mobilization of the peritoneum and a smooth dissection of the psoas muscle. However, the anatomical limits of this technique, as the height of the iliac crest and lumbar plexus morphology below the psoas muscle could create problems in treating some levels (i.e. L4-5) thus limiting the applications of this technique. Huge X-rays exposition is another issue correlated with this technique, because fluoroscopy is mandatory for the correct identification of landmarks and positioning of the hardware and in each step of the procedure. A good option for an anterior approach to the lumbar spine is the anterior retroperitoneal approach, useful to perform a lumbar interbody fusion (Anterior Lumbar Interbody Fusion, ALIF) or a Total Disc Replacement (TDR) [1]. This approach is really minimal invasive for the patient, as the only incisions that are required are those of the skin, the fatty under skin layer and the fascia of the rectus muscles. From there beyond, only the smooth blunt dissection and mobilization of the rectus muscle and of the abdominal structures are required to reach the intervertebral disc. In this paper we will describe the technique we personally use to perform the retroperitoneal anterior approach; it's a video-assisted technique with the use of an endoscope. This tool offers a good magnification and illumination of the surgical field, minimizing the incision and tissue damage and further increasing the precision of the surgery. The use of endoscope improves visualization of spinal anatomy, allowing a complete disc removal. Endoscopic disc decompressions are performed under continuous visualization.

\section{Retroperitoneal Anterior Approach to the Lumbar Spine}

The retroperitoneal approach is our preferred technique because it respects the anatomy of the abdominal structures. The standard retroperitoneal approach is performed with a midline skin and under skin layer incision, with a left approach to the intra-abdominal structures. Different authors have proposed many variations to this technique. In 2012 a midline approach from the right side was proposed by Edgard-Rosa et al. [2]. In this paper the authors proposed a new mobilization technique of the vena cava to perform interbody fusions from L2 to L5, with an approach below the aortic bifurcation to L5-S1 disc. The authors reported four major venous injuries on a total of 469 patients included in the study. However, we prefer the left side approach because the first big vessel found during the approach is an artery (the common iliac artery). This decreases the risk of vascular injury because of the thicker walls of arteries in respect to veins. Authors preferred technique is performed with the patient lying in a supine position, with extended legs. A 3 - $5 \mathrm{~cm}$ transversal medial incision (for L5-S1 exposure) or a longitudinal left paramedian incision (for L4-L5 exposure) is performed (Figure 1). The anterior sheath of the rectus is cut and the muscle is retracted upward and laterally, to preserve nervous and bloody supply avoiding damages to the inferior epigastric vessels; the peritoneum and the left ureter are then gently pushed from the left to the right side (Figure 2) until the psoas muscle is seen. At this point the arcuate ligament is cut (Figure 3). This allows the widening of the working space and a better visualization of the deep structures. After the insertion of deep retractors, the big vessels are visualized and mobilized. For the L4-5 disc, aorta, cava and common iliac veins and arteries are retracted laterally from left to right after the ligature of the ileolumbar veins. The L5-S1 disc is located below aorta and cava bifurcation into the two common iliac arteries and veins, respectively. These vessels are then retracted on both sides to expose the disc, and the middle sacral vessels are coagulated with a bipolar forceps or ligated.

After the complete exposure of the disc, the retractors are fixed to the bony surface through dedicated pins. This very stable configuration avoids the risk of soft tissue or vascular injury due to the implant of the pins. A ring is then placed to connect the handles of the retractors, in order to achieve a 360 degrees stability to keep the 


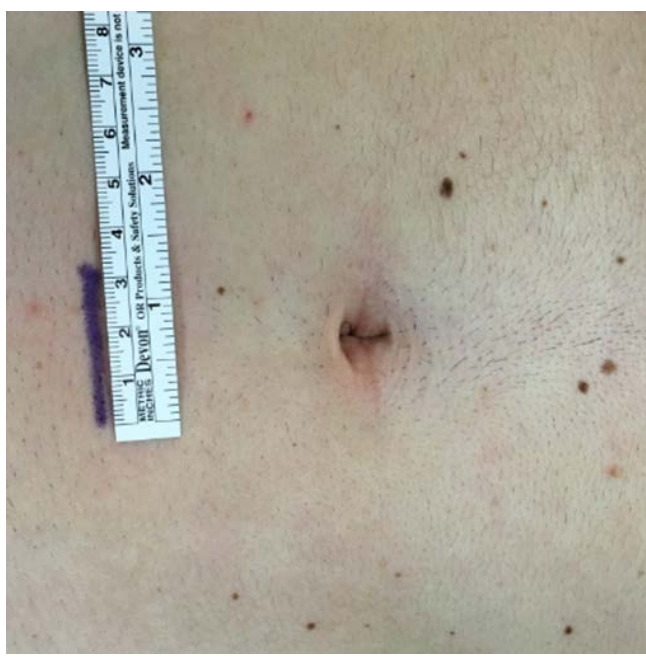

Figure 1. Midlineskin incision below the navel.

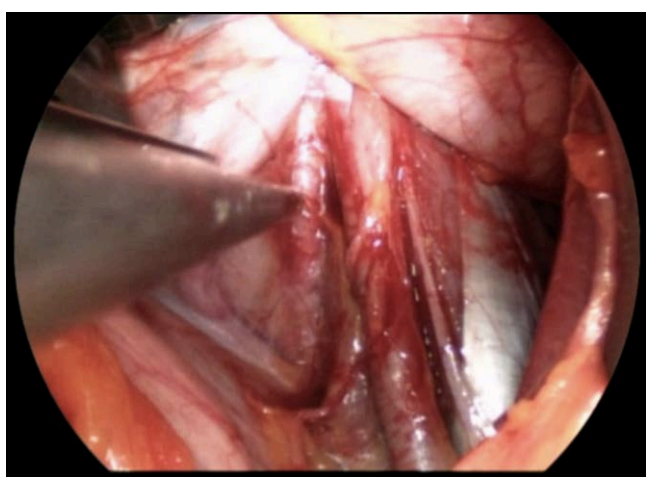

Figure 2. Intraoperative endoscopic view of ureter (indicated with an anatomic claw) and common iliac artery (on the right).

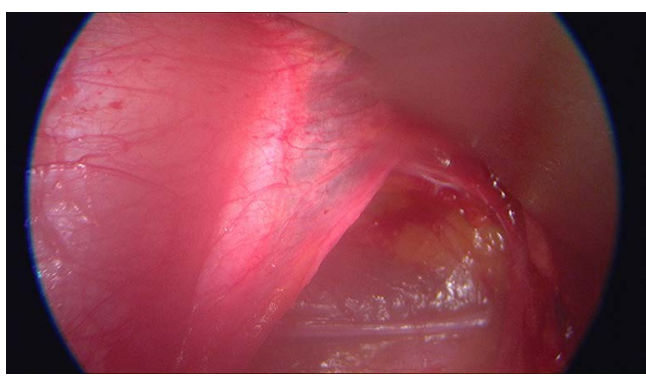

Figure 3. Intraoperative endoscopic view of the arcuate line (also known as linea semicircularis or Douglas' line).

surgical field wide open. Under endoscopic view and magnification, two horizontal cuts are performed with a scalpel on the anterior longitudinal ligament, corresponding to the upper and lower limits of the disc. A vertical incision is then performed on the midline, and the anterior ligament is detached from the midline laterally. This procedure results in two flaps that are preserved to be sutured together after the cage implant. The disc is then removed and the endplates prepared scrapping off the cartilaginous layer (Figure 4). Increasing sizes templates are then implanted in the intervertebral space, in order to find the proper fit (Figure 5). Finally, the definitive cage is implanted and fixed with an anterior plate with screws (we usually implant a three-screw plate for L5-S1 
space, and a four-screw plate for the L4-5 disc) in order to increase primary stability (Figure 6). It has been demonstrated that a cage with an anterior plate has the same stiffness than a cage with posterior fixation (four pedicle screws) [3] (Figure 7). The whole procedure is performed through a $5 \mathrm{~cm}$ incision with the use of an endoscopic camera. This allows the best view on a HD monitor at a higher magnification for all the surgical steps, allowing a lesser invasivity and a better accuracy.

Anterior retroperitoneal approach allows the treatment of the lumbar spine preserving the paraspinal muscles and consequently without disruption of the posterior tension band. Moreover, joint morphology and innervation are preserved, maintaining a better proprioceptive capacity at the treated levels. Furthermore, performing a single anterior approach decreases the morbidity of the patients, and the implant of a wider intervertebral cage is possible without entering the canal. This allows a better restoration of the segmental lordosis and a greater cage-bone contact surface, promoting osteointegration and fusion. Finally, this kind of approach requires only one shot with the C-arm to evaluate the final position of the cage. Since the whole procedure is observed directly

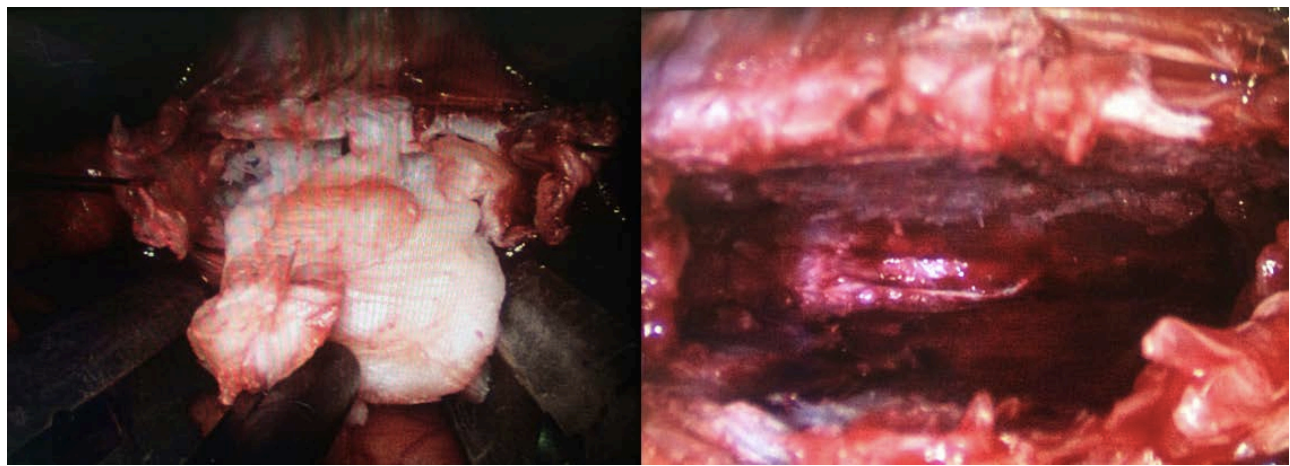

Figure 4. Intraoperative endoscopic view of discectomy and disc space preparation.

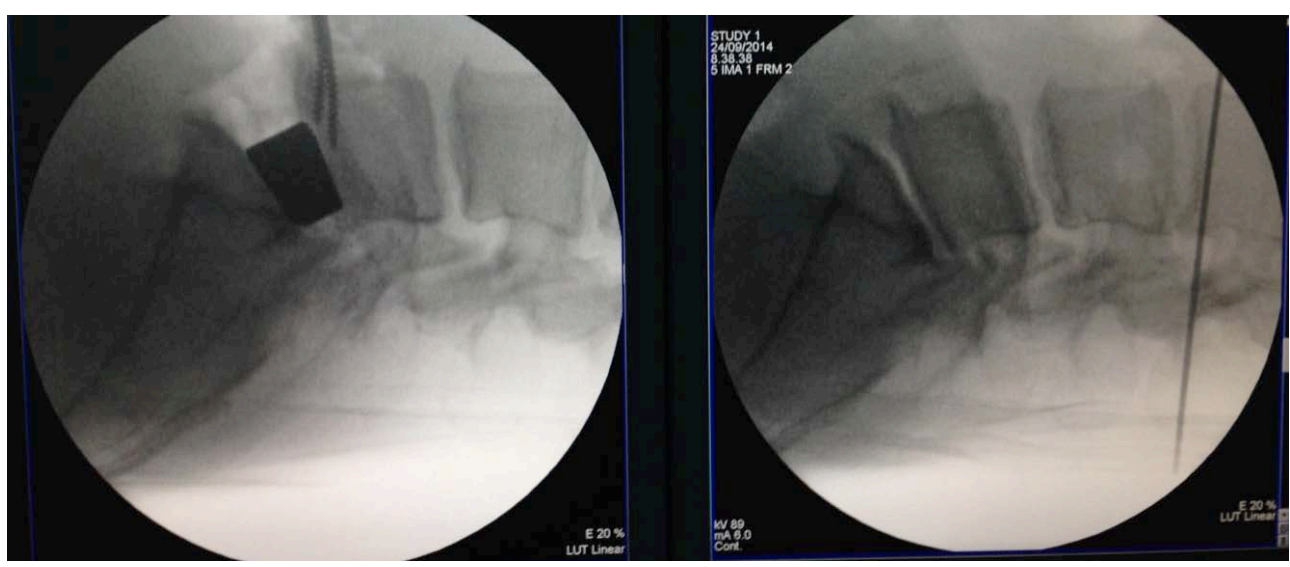

Figure 5. Intraoperative X-ray showing the increase of lumbar lordosis after cage implant.

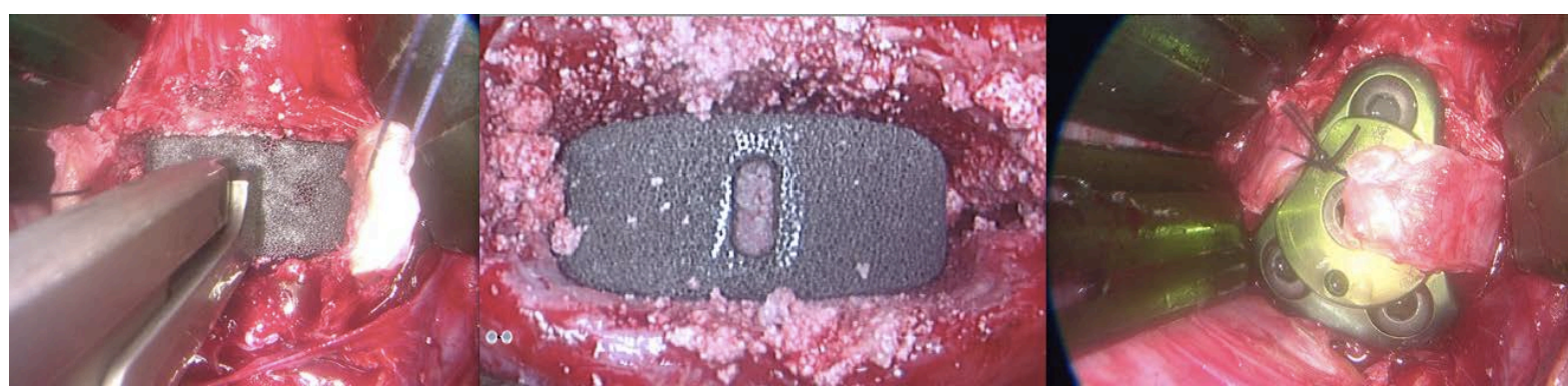

Figure 6. Intraoperative endoscopic view of definitive trabecular metal cage filledwith autologous bone graft and subsequent anterior plate fixation. The two flaps of the anterior longitudinal ligament can now be sutured together. 


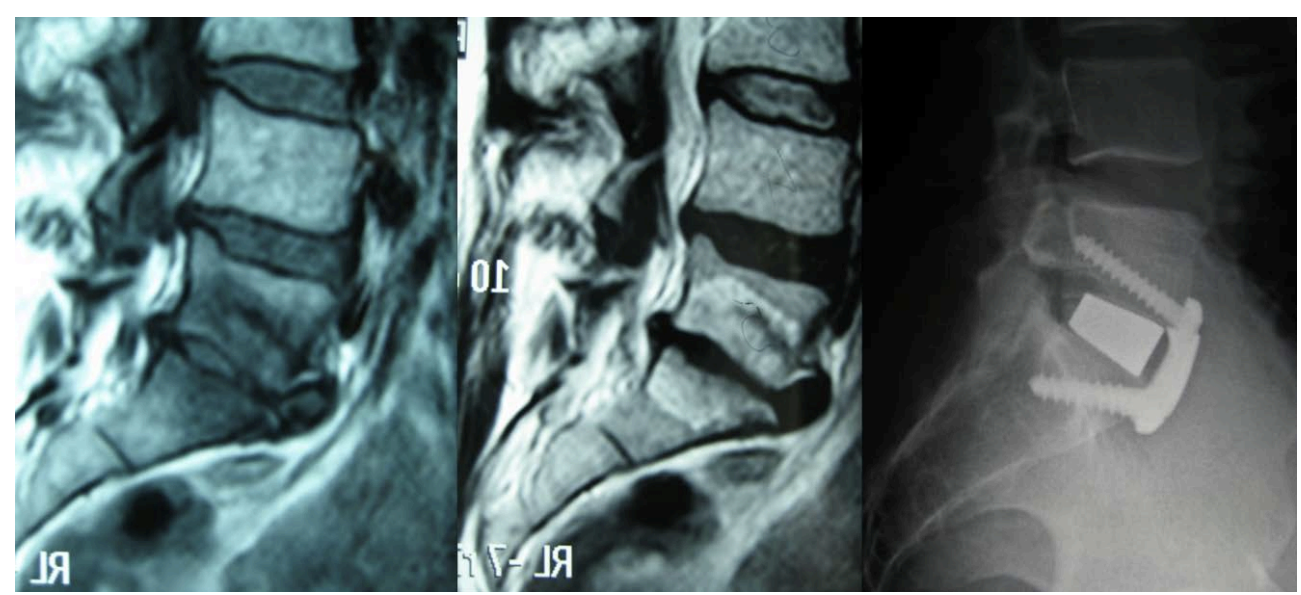

Figure 7. Preoperative sagittal lumbar MRI (T1 and T2 sequences) showing a L5-S1 discopathy (Pfirrmann 5) and postoperative X-ray (lateral view) of a L5-S1 ALIF. The patient has been treated with a tantallum cage and a three-screw anterior plate. This construct has the same stability than an anterior cage with four pedicle screws. It is clearly evaluable how the segmental lordosis have been properly increased after surgery.

from the surgeon and using the endoscope, no intermediate X-rays are needed. This decreases the radiation dose to the patient and to the surgeons in respect to standard posterior procedures, in which fluoroscopy could be performed for each pedicle screw and cage insertion.

\section{Complications}

The anterior retroperitoneal approach allows the treatment of a wide range of pathologies affecting the lumbar spine, but can be a source of hazardous complications. The most common are: damages to the vascular, urologic, gastrointestinal and neurologic structures. For these reasons, many spinal surgeons need the help of vascular or general surgeons (access surgeon) to perform this procedure.

\subsection{Vascular Complications}

Mobilization of iliac vessels is a cause of potential lesions. The reported incidence of major vascular injury varies in literature between $2 \%$ and $4 \%$, depending on the studies [4]-[10]. The left common iliac vein is the most likely vascular structure to be injured during anterior retroperitoneal approach, especially for the L4-5 disc. Calcified arteries in elderly could represent an increased risk of damage during vessel manipulation. Postoperative bleeding could occur when an incomplete ligature of the ascending ileolumbar vein has been performed during the exposition of L4-5 disc or due to a missed identification of small bleeding arteries. An adequate check should be performed before closure with an increasing blood pressure of the patient. Apart from intraoperative or postoperative bleeding and risk of massive and life threating hemorrhage, venous thrombotic occlusion could occur in the postoperative period. This could happen as a consequence of a prolonged retraction of the vessels. Arterial thrombosis occurs in less than $1 \%$ of cases, and is frequently associated with prolonged retraction [5] [6] or due to pathologic calcifications.

\subsection{Urologic Complications}

The ureter is the main extra peritoneal urologic structure observed during the exposure. It is usually attached to the inferior surface of the peritoneum, and is clearly visible when laterally retracted. It should be pinched with an anatomical claw to be identified observing its typical contraction. Ureteral lesions during primary anterior surgery are really rare, while they happen more frequently during revision surgery, as the scar tissue in front of the instrumentation could encase it [11]. Retrograde ejaculation is a complication that couldoccur in men underwent anterior retroperitoneal approaches, especially to L5-S1. Sterility could result from the inability of the internal vescical sphincter that allows the semen to enter the bladder instead of traveling up the urethra. The superior hypogastric plexus provides innervation to the internal vescical sphincter. This plexus lies on the anterior 
surface of the L5-S1 disc, and needs to be mobilized to perform the discectomy. To reduce the risk of this complication it is important to avoid using monopolar coagulation during the deep dissection. The overall rate of retrograde ejaculation varies from $0.42 \%$ to $5.9 \%$, according to the literature [12] [13]. A study of Sasso et al. published in 2003 [14] demonstrated that patients underwent a lumbar discectomy and fusion through a transperitoneal approach at L4-5 and L5-S1 levels had a 10-times higher incidence of retrograde ejaculation than those who had the same procedures with a retroperitoneal approach.

\subsection{Gastrointestinal Complications}

The most common gastrointestinal complication observed in the postoperative period in patients underwent an anterior retroperitoneal approach is ileus [11]. The use of anorogastric tube and preoperative bowel preparation are routine measures to decrease the risk of this complication. All the patients are provided with $140 \mathrm{mg}$ of macrogol oral powder in 2 liters of water the day before surgery. The postoperative use of opioids could also increase the risk of constipation.

\section{Personal Experience}

Analyzing the patients underwent an anterior retroperitoneal lumbar access in our Department from 2010 to 2012, we obtained a cluster of 163 patients, 60 males and 103 females. The mean age was $43 \pm 10$ years for men and $46 \pm 9$ years for women. A single level L5-S1 anterior approach was performed in 141 patients (61 men 43.3\% and 80 women 56.7\%), a single level L4-L5 anterior approach was performed in 12 patients (3 men 25\%, 9 women 75\%), a two-level L4-L5 L5-S1 was performed in 10 female patients. ALIF was performed in a total of 132 patients (81.0\%). A disc prosthesis was implanted in 10 patients (6.1\%). An ALIF + TDR was performed in 4 patients (2.5\%). 17 patients (10.4\%) underwent a double approach surgery in a single stage. If we consider only the single level procedures (L4-5 or L5-S1), 139 patients can be enrolled. The average time of surgery was 116.4 minutes, with $156.1 \mathrm{ml}$ of intraoperative blood loss. These values suggest that anterior approach for single level treatment is a quick procedure with low intraoperative blood loss. If we further consider our rate of complications in the same cluster, we had 3 cases of retrograde ejaculation (2.16\%), 2 iliac vein lesions (1.44\%) and one immediate revision for hardware malposition (0.72\%) (Table 1$)$.

\section{Conclusion}

The anterior approach to the lumbar spine is an effective technique to treat disc degenerative disease, spinal deformities, traumas, tumors or infections. Though being a fast procedure that is becoming more often used by spinal surgeons, the anterior approach requires an adequate knowledge of the anatomy of the abdomen to lessen the risks of intraoperative complications. The retroperitoneal approach has been demonstrated to be safe and less risky than the transperitoneal one, especially regarding the retrograde ejaculation complication [14]. What we suggest and usually perform is a retroperitoneal video-assisted technique that allows a better view with the use of an endoscopic camera, allowing a magnification of the approach-related structures on a monitor. This let the surgeon being less invasive and more accurate in performing this approach and the discectomy for an ALIF or a TDR procedure. In our personal experience we observed how this technique is fast with a very low blood loss in respect to traditional posterior techniques. In our series the rate of complications is lower or similar to those found in literature. Video-assisted anterior lumbar surgery has advantages of little incision, minimal tissue

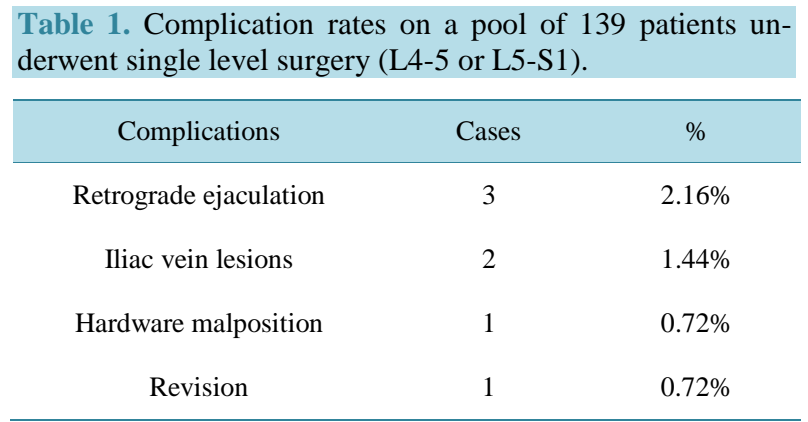


retraction and minimal bleeding with consequent less blood loss, less trauma. Endoscopic video-assisted surgery is a safe, effective method in treating degenerative disease of the lumbar spine. Although, in literature there is no evidence of difference in meanoperative room time, intraoperative complications, or total hospital length of stay, between video assisted and traditionalsurgery [15].

\section{References}

[1] Bassani, R., Sinigaglia, A. and Lamartina, C. (2001) Video-Assisted Minimally Invasive Lumbartotal Disc Replacement. European Spine Journal, 20, 2282-2283. http://dx.doi.org/10.1007/s00586-011-2077-y

[2] Edgard-Rosa, G., Geneste, G., Nègre, G. and Marnay, T. (2012) Midline Anterior Approach from the Right Side to the Lumbar Spine for Interbody Fusion and Total Disc Replacement. Spine, 37, E562-E569. http://dx.doi.org/10.1097/brs.0b013e31823a0a87

[3] Nicolas, T.A., Yantzer, B.K., Alameda, S., Johnson, W.M. and Guiot, B.H. (2007) Augmentation of an Anterior Lumbar Interbody Fusion with an Anteriorplate or Pedicle Screw Fixation: A Comparative Biomechanical in Vitro Study. Journal of Neurosurgery Spine, 6, 267-271. http://dx.doi.org/10.3171/spi.2007.6.3.267

[4] Fantini, G.A., Pappou, I.P., Girardi, F.P., Sandhu, H.S. and Cammisa Jr., F.P. (2007) Major Vascular Injury during Anterior Lumbar Spinal Surgery. Incidence, Risk Factors, and Management. Spine, 32, 2751-2758. http://dx.doi.org/10.1097/BRS.0b013e31815a996e

[5] Brau, S.A., Delamarter, R.B., Schiffman, M.L., Williams, L.A. and Watkins, R.G. (2004) Vascular Injury during Anterior Lumbar Surgery. Spine Journal, 4, 409-412. http://dx.doi.org/10.1016/j.spinee.2003.12.003

[6] Kulkarni, S.S., Lowery, G.L., Ross, R.E., Ravi Sankar, K. and Lykomitros, V. (2003) Arterial Complications Following Anterior Lumbar Interbody Fusion: Report of Eight Cases. European Spine Journal, 12, 48.

[7] Gumbs, A.A., Shah, R.V., Yue, J.J. and Sumpio, B. (2005) The Open Anterior Paramedian Retroperitoneal Approach for Spine Procedures. Archives of Surgery, 140, 339-343. http://dx.doi.org/10.1001/archsurg.140.4.339

[8] Fritzell, P., Hägg, O. and Nordwall, A. (2003) Complications in Lumbar Fusion Surgery for Chronic Low Back Pain: Comparison of Three Surgical Techniques Used in a Prospective Randomized Study. A Report from the Swedish Lumbar Spine Study Group. European Spine Journal, 12, 178-189.

[9] Holt, R.T., Majd, M.E., Vadhva, M. and Castro, F.P. (2003) The Efficacy of Anterior Spine Exposure by an Orthopedic Surgeon. Journal of Spinal Disorders \& Techniques, 16, 477-486. http://dx.doi.org/10.1097/00024720-200310000-00007

[10] Kaiser, M.G., Haid, R.W., Subach, B.R., Miller, J.S., Smith, C.D. and Rodts, G.E. (2002) Comparison of the Mini Open versus Laparoscopic Approach for Anterior Lumbar Interbody Fusion: A Retrospective Review. Neurosurgery, 51, 97-103. http://dx.doi.org/10.1097/00006123-200207000-00015

[11] Fantini, G.A. and Pawar, A.Y. (2013) Access Related Complications during Anterior Exposure of the Lumbar Spine. World Journal of Orthopaedics, 4, 19-23. http://dx.doi.org/10.5312/wjo.v4.i1.19

[12] Flynn, J.C. and Price, C.T. (1984) Sexual Complications of Anterior Fusion of the Lumbar Spine. Spine, 9, 489-492. http://dx.doi.org/10.1097/00007632-198407000-00013

[13] Tiusanen, H., Seitsalo, S., Osterman, K., et al. (1995) Retrograde Ejaculation after Anterior Interbody Fusion. European Spine Journal, 4, 339-342. http://dx.doi.org/10.1007/BF00300293

[14] Sasso, R.C., Burkus, J.K. and LeHuec, J.C. (2003) Retrograde Ejaculation after Anterior Lumbar Interbody Fusion. Transperitoneal versus Retroperitoneal Exposure. Spine, 28, 1023-1026. http://dx.doi.org/10.1097/01.BRS.0000062965.47779.EB

[15] Shirzadi, A., Mukherjee, D., Drazin, D.G., Paff, M., Perri, B., Mamelak, A.N. and Siddique, K. (2012) Use of the Video Telescopeoperating Monitor (VITOM) as an Alternative to the Operating Microscope in Spine Surgery. Spine, 37, E1517-E1523. http://dx.doi.org/10.1097/BRS.0b013e3182709cef 